\title{
Grenzen der arthroskopischen Behandlung degenerativer Läsionen des ulnokarpalen Komplexes in Abhängigkeit der Ulnavarianz
}

\section{Limits to Arthroscopic Treatment of Degenerative Triangular Fibrocartilage Complex Lesions Depending on the Ulnar Variance}

\author{
Autoren

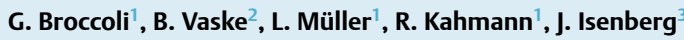 \\ Institute \\ ${ }^{1}$ Plastische, Ästhetische und Handchirurgie, KRH-Klinikum Nordstadt, Hannover \\ ${ }^{2}$ Institut für Biometrie, Medizinische Hochschule Hannover, Hannover \\ ${ }^{3}$ Unfallchirurgie und Orthopädie, KRH-Klinikum Nordstadt, Hannover
}

Schlüsselwörter

- ulnokarpaler Komplex

- Handgelenksarthroskopie

- Ulna-Impaktion-Syndrom

- Ulnavarianz

- Ulnaverkürzungsosteotomie

Key words

- triangular fibrocartilage complex

- wrist arthroscopy

- ulnar impaction syndrome

- ulnar variance

- ulnar shortening osteotomy

eingereicht $\quad 5.7 .2013$

akzeptiert $\quad$ 28.2.2014

Bibliografie

DOI http://dx.doi.org/

10.1055/s-0034-1371851

Handchir Mikrochir Plast Chir

2014; 46: 151-162

(c) Georg Thieme Verlag KG

Stuttgart · New York

ISSN 0722-1819

Korrespondenzadresse

\section{Giuseppe Broccoli}

Plastische, Ästhetische und Handchirurgie

KRH-Klinikum Nordstadt

Halltenhoffstraße 41

30167 Hannover

giubro@gmx.de

\section{Zusammenfassung}

$\nabla$

Ziel: Die vorliegende prospektive Studie untersucht den Einfluss der statischen Ulnavarianz auf den Erfolg des arthroskopischen Débridements einer degenerativen TFCC-Läsion.

Patienten und Methode: 10 Patienten mit einer Ulna-plus-Varianz („Ulna + “) und 12 Patienten mit einer Ulna-null- oder Ulna-minus-Varianz („Ulna-/0“) wurden präoperativ (U0) sowie 2 (U2) und 6 (U6) Monate nach arthroskopischem Débridement einer degenerativen TFCC-Läsion untersucht und miteinander verglichen, wobei bei 9 der 10 Patienten mit einer Ulna-plus-Variante aufgrund anhaltender Beschwerden nach der U2-Untersuchung eine Ulnaverkürzungsosteotomie (UVO) erfolgt war. Folgende Parameter wurden jeweils erfasst: Schmerzen in Ruhe und bei Belastung, die summierte Handgelenksbeweglichkeit aus Extension/Flexion, Radial-/Ulnarduktion sowie Pro-/Supination im Vergleich zur gesunden Gegenseite, die Kraft der betroffenen Hand im Vergleich zur gesunden Gegenseite, der Mayo Modified Wrist Score (MMWS), der Krimmer-Score sowie der DASH-Score. Zum Zeitpunkt UO fanden sich zwischen den beiden Kohorten „Ulna-/0“ und „Ulna + “ bis auf ein Merkmal („Ruheschmerz“) keine signifikanten Unterschiede.

Ergebnisse: Die Ergebnisse in der Kohorte „Ulna + “ verharrten zum Zeitpunkt U2 auf dem signifikant oder tendenziell schlechteren Niveau im Vergleich zur Kohorte „Ulna-/0“. Die anschließende operative Behandlung der Kohorte „Ulna + “ mittels UVO führte zur fast vollständigen Angleichung aller Ergebnisse zum Zeitpunkt U6. Darüber hinaus fanden sich 6 Monate postoperativ innerhalb der jeweiligen Kohorte tendenzielle bzw. signifikante Verbesserungen der Erhebungsmerkmale gegenüber der Situation U0. Zum Zeitpunkt U6 waren noch 4 Patienten des Gesamtkollektivs arbeitsunfähig.

\section{Abstract}

$\nabla$

Purpose: The present prospective study investigated the influence of the static ulnar variance on the success of arthroscopic debridement of degenerative TFCC lesions.

Patients and Methods: 10 patients with an ulnar positive variance ("Ulna+") and 12 patients with ulnar neutral or ulnar negative variance ("Ulna-/0") were examined preoperatively (U0), as well as at 2 (U2) and 6 (U6) months after arthroscopic debridement of degenerative TFCC lesions and compared with each other. After the U2 investigation due to persistent complaints in 9 of 10 patients with an ulnar positive variance there was a need for further surgery, consisting of ulnar shortening osteotomy (USO). The following parameters were recorded in each case: pain at rest and with load, the summed wrist range of motion - consisting of extension and flexion, radial and ulnar deviation, pronation and supination - compared to the contralateral side, the strength of the affected hand compared to the contralateral side, the Mayo modified wrist score (MMWS), the Krimmer score and the DASH score. Preoperatively there were no significant differences between the 2 cohorts "Ulna + " and "Ulna-/0" except for the characteristic "pain at rest".

Results: At 2 months postoperatively (U2), the results in the cohort "Ulna + " remained at a significantly or tendentially poorer level compared to the cohort "Ulna-/0". The subsequent surgical treatment of the subgroup "Ulna + " with USO led to almost complete approximation of the results at 6 months postoperatively (U6). In addition to this, with time (U6) within each subgroup there were tendential or significant improvements of all characteristics compared to the preoperative situation (U0). At U6 four of 22 patients were unable to work.

Conclusion: Degenerative lesions of the TFCC can be treated successfully by arthroscopic debri- 
Schlussfolgerung: Degenerative Läsionen des TFCC können im Falle einer Ulna-minus- und Ulna-null-Variante durch ein arthroskopisches Débridement erfolgreich behandelt werden. Patienten mit Ulna-plus-Varianz können bei Beschwerdepersistenz nach Débridement des TFCC sekundär erfolgreich mit einer Ulnaverkürzung behandelt werden. dement in cases of ulnar negative and ulnar neutral variance. Patients with ulnar positive variance and persistent complaints after debridement of the TFCC can be treated successfully with a secondary ulnar shortening osteotomy.

\section{Einleitung}

$\nabla$

Ein häufiger Grund für den ellenseitigen Handgelenksschmerz stellt der Verschleiß bedingte (degenerative) Schaden des ulnokarpalen Komplexes (triangular fibrocartilage complex, TFCC) dar [1-4]. Häufig, aber nicht immer, ist die Ursache hierfür eine anlagebedingte (idiopathische) oder posttraumatische Ulnaplus-Variante [1,3-9]. Zur Behandlung Verschleiß bedingter TFCC-Läsionen werden bei Versagen konservativer Therapiemaßnahmen zur Druckentlastung des ulnokarpalen Gelenkkompartimentes neben dem alleinigen arthroskopischen Débridement der Läsion des Discus ulnocarpalis intra- und extraartikuläre Osteotomie- und Resektionsverfahren der Ulna additiv oder isoliert sowie simultan oder mehrzeitig ausgeführt [17,10-17]. Mehrere Arbeiten weisen darauf hin, dass einerseits mit zunehmender Ulna-plus-Situation das alleinige arthroskopische Débridement nicht erfolgversprechend ist, andererseits eine Ulnaverkürzungsosteotomie, im Folgenden UVO genannt, die fortbestehenden Beschwerden zumindest zum Teil zu beseitigen vermag $[2,4,5,7,11,12,14,15,18-22]$. Ziel der vorliegenden Studie war es, prospektiv den Einfluss der Ulnavarianz auf den Erfolg eines arthroskopischen Débridements einer degenerativen TFCC-Läsion zu untersuchen. Dabei gingen wir davon aus, dass sich die klinischen Ergebnisse der Patienten mit einer Ulna-plus-Variante von denen der Patienten mit Ulna-minusbis Ulna-null-Variante im Nachuntersuchungszeitraum unterscheiden und sich bei Versagen des arthroskopischen Débridements durch eine zusätzliche UVO annähern [1,3-5, 8, 18, 21,22].

\section{Patienten und Methoden}

$\nabla$

Die prospektive Studie wurde durch die Ethikkommission der Ärztekammer Niedersachsen genehmigt. 35 Patienten willigten in die Teilnahme an der Studie ein. 22 Patienten erfüllten mit einem ulnaren Handgelenksschmerz unter Alltagsbelastung, der sich über 3 Monate unter konservativen Therapiemaßnahmen (temporäre Ruhigstellung, Antiphlogistika- und Analgetikaeinnahme sowie Physiotherapie) als therapierefraktär erwies und arthroskopisch gesicherter, degenerativer TFCC-Läsion den Studieneinschluss. Ausgeschlossen wurden Patienten mit einer traumatischen TFCC-Läsion, der Abwesenheit einer TFCC-Läsion und einem von der sekundären UVO abweichendem Folgeeingriff. Die statische Ulnavarianz wurde bei allen Patienten präoperativ bzw. postoperativ nach zusätzlicher UVO nativ radiologisch anhand eines posterioranterioren Röntgenbildes des betroffenen Handgelenkes in $90^{\circ}$ Abduktion der Schulter sowie $90^{\circ}$ Flexion des Ellenbogens und Neutralrotation des Unterarmes mittels der Methode nach Gelberman digital mit Centricity PACS (Firma GE Healtcare ${ }^{\circledR}$ GmbH, Solingen, Deutschland) bestimmt $[23,24]$. Das Gesamtkollektiv wurde in Abhängigkeit der präoperativen Ulnavarianz in die Kohorten „Ulna + “ (Ulna-plusVarianz) und „Ulna-/0“ (Ulna-minus- bis Ulna-null-Varianz) unterteilt.
Bei 10 Patienten, 5 Frauen und 5 Männer im Alter von 19 bis 61 (Median 44) Jahren, fand sich eine Ulna-plus-Variante von durchschnittlich 3,1 (Min. 1, Max. 5) mm. Das rechte Handgelenk war in 5, das dominante in 8 Fällen betroffen. Arbeitsunfähigkeit bestand bei 8 der 10 Patienten. In der Vorgeschichte fand sich 2-mal ein Beginn der ulnaren Handgelenksbeschwerden nach einem Stauchungs- bzw. Verdrehtrauma des Handgelenkes.

12 Patienten, 4 Frauen und 8 Männer im Alter von 25 bis 68 (Median 46) Jahren, wiesen eine Ulna-null- oder Ulna-minus-Variante von durchschnittlich -0,3 (Min. 0, Max. -1) mm auf. Das rechte Handgelenk war in 9, das dominante in 12 Fällen betroffen. Arbeitsunfähigkeit bestand bei 9 der 12 Patienten. In der Vorgeschichte fand sich 4-mal ein Beginn der ulnaren Handgelenksbeschwerden nach einem Stauchungs- bzw. Verdrehtrauma des Handgelenkes.

Die Arthroskopie erfolgte in allen Fällen ambulant im Aushang $(6,8 \mathrm{~kg})$ unter Verwendung eines Tasthakens über folgende Portale: 3-4, 6R sowie mediokarpal radial und ulnar [25,26]. Läsionen des TFCC ('마 Video 1) wurden nach Palmer klassifiziert, Knorpelschäden analog der Outerbridge-Einteilung und Läsionen des lunotriquetralen Bandes analog der Klassifikation für skapholunäre Bandverletzungen nach Geissler [9,27,28]. Das Débridement des TFCC erfolgte mittels Punch und motorbetriebenem Shaver. Die häufigste Pathologie des TFCC war dem Typ 2D nach Palmer zuzuordnen $(\mathrm{n}=11) ; 8$-mal fand sie sich in der Kohorte „Ulna + “. Die 2C-Läsion war am zweithäufigsten vertreten $(n=8)$; 7-mal war sie in der Kohorte „Ulna-/0“ anzutreffen. In beiden Kohorten wurde je eine $2 \mathrm{E}$-Läsion verzeichnet. Nur in einem Fall bestand eine Palmer $2 \mathrm{~A}$-Läsion, die der Kohorte „Ulna-/0“ zuzuordnen war. Darüber hinaus fanden sich eine ulnokarpale Chondromalazie I ${ }^{\circ}$ einmal, II $^{\circ}$ 9-mal, III $^{\circ}$ 4-mal und $\mathrm{IV}^{\circ} 8$-mal. Eine Partialruptur des lunotriquetralen Bandappara-

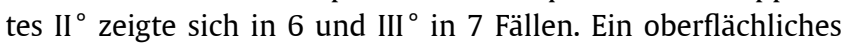
Débridement des ulnokarpalen Komplexes erfolgte in einem Fall, eine Partialresektion mit Entfernung instabiler und flottierender Anteile des Discus ulnocarpalis wurde bei 21 Patienten durchgeführt. Die arthroskopischen Maßnahmen am TFCC wurden regelhaft durch eine partielle Synovektomie $(n=22)$ und eine ulnokarpale Chondroplastik $(n=21)$ ergänzt. Aufgefaserte oder zerrissene Anteile des lunotriquetralen Bandes wurden in 13 Fällen débridiert. Postoperativ wurde das Handgelenk in allen Fällen für eine Woche ruhiggestellt, gefolgt von einem schmerzadaptierten Belastungsaufbau unter physiotherapeutischer Anleitung. Des Weiteren wurde die Einnahme von Antiphlogistika (Ibuprofen $600 \mathrm{mg} 1-1-1$ ) verordnet. Befunde und Maßnahmen sind in $\bullet$ Tab. 1 zusammengefasst.

Präoperativ (U0) sowie 2 und 6 Monate postoperativ (U2, U6) wurden die folgenden Parameter erfasst:

- ulnokarpaler Schmerz in Ruhe bzw. bei Belastung des Handgelenkes (maximaler Grobgriff) auf der visuellen Analogskala (VAS) von 0 bis 10, wobei „0“ kein Schmerz und „10“ maximaler Schmerz bedeutet,

- die relative summierte Handgelenksbeweglichkeit aus Extension/Flexion, Radial-/Ulnarduktion sowie Pronation/Supination 


\begin{tabular}{|c|c|c|c|}
\hline Beobachtungsparameter & „Ulna+“ $(n=10)$ & „Ulna-/0“ (n=12) & $\mathbf{p}$ \\
\hline Alter (Jahren)* & $43,2 \pm 14,2$ & $46,9 \pm 12,6$ & $>0,05$ \\
\hline männlich & 5 & 8 & $>0,05$ \\
\hline weiblich & 5 & 4 & $>0,05$ \\
\hline betroffene Seite: rechts & 5 & 9 & $>0,05$ \\
\hline betroffene Seite: dominant & 8 & 12 & $>0,05$ \\
\hline Trauma in der Vorgeschichte & 2 & 4 & $>0,05$ \\
\hline Dauer der Beschwerden (Wochen)* & $31 \pm 20,1$ & $35,9 \pm 41,4$ & $>0,05$ \\
\hline Arbeitsunfähigkeit (U0) & 8 & 9 & $>0,05$ \\
\hline Arbeitsunfähigkeit (U2) & 8 & 2 & $<0,01$ \\
\hline Arbeitsunfähigkeit (U6) & 2 & 2 & $>0,05$ \\
\hline präoperative Ulnavarianz (mm) & 3,1 (Min. 1, Max. 5) & $-0,3$ (Min. 0, Max. -1 ) & $<0,001$ \\
\hline Arthroskopie & 10 & 12 & $>0,05$ \\
\hline \multicolumn{4}{|l|}{ arthroskopische Befunde } \\
\hline - Discusperforation (Palmer 2C-2E) & 10 & 11 & $>0,05$ \\
\hline - ulnokarpale Chondromalazie & 10 & 11 & $>0,05$ \\
\hline - lunotriquetrale Bandruptur & 9 & 4 & $<0,01$ \\
\hline \multicolumn{4}{|l|}{ arthroskopische Therapie } \\
\hline - partielle Synovektomie & 10 & 12 & $>0,05$ \\
\hline - Discusteilresektion & 10 & 11 & $>0,05$ \\
\hline - lunotriquetrales Banddébridement & 9 & 4 & $<0,01$ \\
\hline - Chondroplastik & 10 & 11 & $>0,05$ \\
\hline Ulnaverkürzungsosteotomie & 9 & 0 & $<0,001$ \\
\hline Dauer bis zur Ulnaverkürzung nach Arthroskopie (Tage)* & $73,1 \pm 37,0$ & - & - \\
\hline postoperative Ulnavarianz (mm) & $-0,2$ (Min. 1, Max. -2 ) & - & - \\
\hline Ausmaß der Ulnaverkürzung $(\mathrm{mm})^{*}$ & $3,6 \pm 2,0$ & - & - \\
\hline
\end{tabular}

Tab. 1 Vergleich der Patienten mit Ulna-plus- bzw. Ulna-nullund Ulna-minus-Varianz: demografische Daten, arthroskopische Befunde, operative Maßnahmen. im Vergleich zur gesunden Gegenseite („Total_ROM_\%“, Absolutwerte nach der Neutral-Null-Methode mittels Goniometer ermittelt, Relativwerte in Prozent),

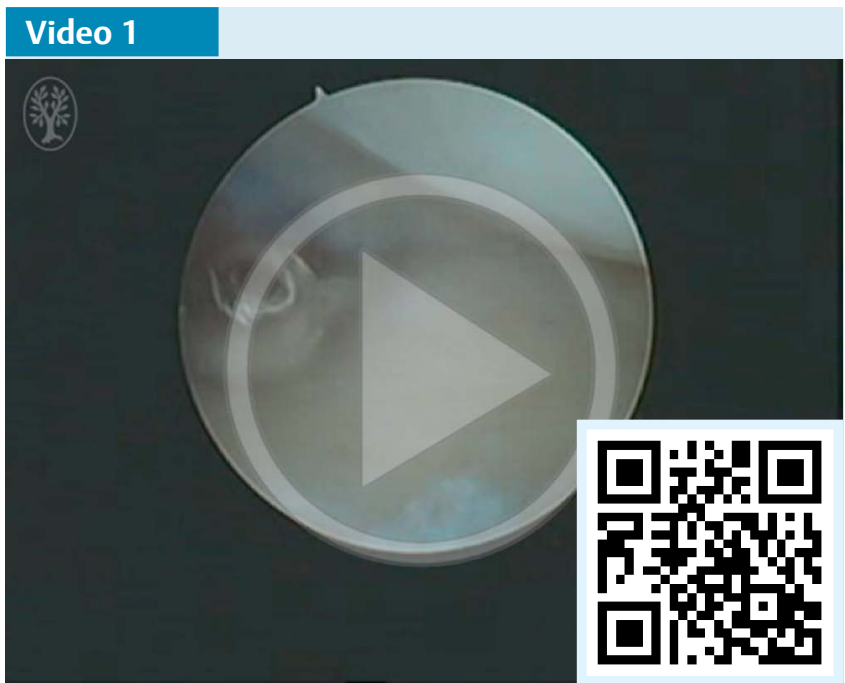

Arthroskopie des linken Handgelenkes: Sicht vom 3-4 - Portal in das ulnokarpale Kompartiment, Instrumentierung (Tasthaken, Klemmchen, motorisierter Shaver) über das 6R Arbeitsportal. Die randständig aufgefaserte, zentroradiale Discus triangularis - Perforation und die korrespondierende, aufgefaserte, lunotriquetrale Bandruptur der Palmer 2D-Läsion werden zunächst mit dem Tasthaken untersucht und nach einer intermittierenden Weitung des 6R - Portals mit dem gebogenen Klemmchen erfolgt das anschließende Débridement der Discus ulnocarpalis-Läsion und der lunotriquetralen Bandläsion mit dem Shaver.

Dieses Video finden Sie auch im Beitrag unter http://dx.doi. org/10.1055/s-0034-1371851.
- die relative Kraft der betroffenen Hand im Vergleich zur gesunden Gegenseite (Messung mittels Mannerfelt-Ulrich intrinisc meter ${ }^{\circledR}$, Ulrich GmbH \& Co. KG, Ulm, Deutschland; Absolutwerte in Einheiten (100 Einheiten = 23 Newton), ,Kraft\%“= Relativwerte in Prozent)

- die Handgelenks- und Handfunktion mittels Mayo Modified Wrist Score (MMWS, „Mayo“) und Handgelenkbewertungsschema nach Krimmer („Krimmer“),

- die Lebensqualität bzw. Alltagseinschränkung mithilfe des DASH-Score („DASH“).

9 Patienten mit Ulna-plus-Variante (2-mal posttraumatisch; $\bullet$ Abb. 1a) sahen aufgrund persistierender Beschwerden zum Zeitpunkt U2 die Notwendigkeit eines Folgeeingriffs gegeben. Bei ihnen wurde im Mittel 73 (Min. 27, Max. 148) Tage nach der Handgelenksspiegelung eine UVO mit doppelter Querosteotomie am Übergang vom distalen zum mittleren Schaftdrittel und dynamischer 6- oder 7-Loch-Kompressionsplattenosteosynthese (Stryker ${ }^{\circledR}$ Trauma AG, Selzach, Schweiz; $\bullet$ Abb. 1b) durchgeführt. Dabei wurde neben dem geplanten Resektionsausmaß der Ulna (angestrebte Ulna-null- bis Ulna-minus-Varianz von - $1 \mathrm{~mm}$ ) die Konfiguration des distalen Radioulnargelenkes (DRUG) nach Tolat berücksichtigt, um eine Inkongruenz des DRUG zu vermeiden [29] ( $\bullet$ Abb. 1a). 2-mal bestand eine schräge Gelenkflächenanordnung des DRUG vom Typ II nach Tolat, 3-mal zeigte sich eine invers schräge Gelenkflächenanordnung vom Typ III nach Tolat sowie 4-mal bestand eine parallele Gelenkflächenkonfiguration vom Typ I.

\section{Statistik}

$\nabla$

Die deskriptive Statistik wurde bei nachgewiesener Normalverteilung aller erhobenen Werte mittels des Kolmogorov-Smirnov Tests $(p>0,05)$ mit parametrischen Verfahren mittels IBM $^{\circledR}$ SPSS $^{\circledR}$ Statistics (Version 19, IBM Corp., Armonk, NY, USA) er- 

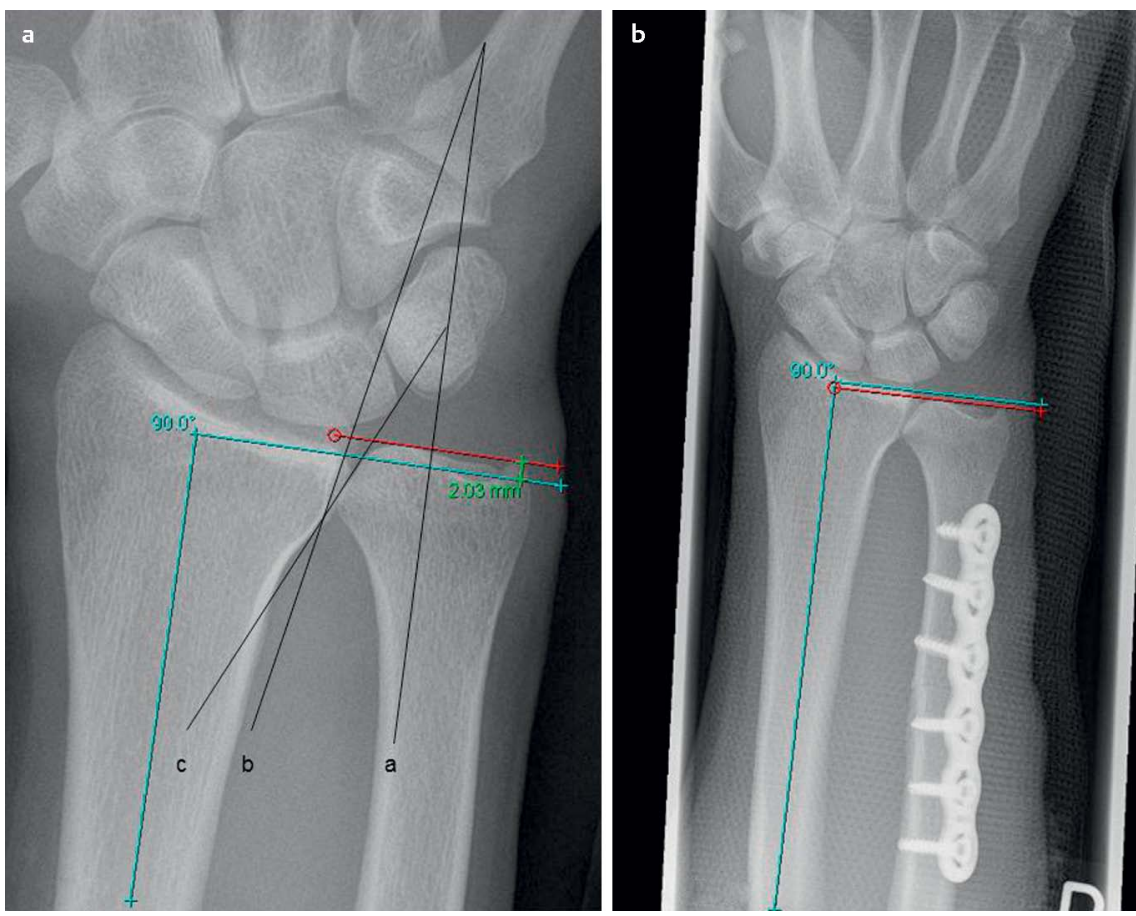

Abb. 1 a, b Radiologische Bestimmung der präoperativen Ulnavarianz im posterioranterioren Strahlengang des rechten Handgelenkes nach Gelberman [23] (Abb. 1a), türkis: Lotrechte auf die Radiusschaftlängsachse, rot: Parallele zur Radiusschaftlotrechten auf Höhe der distalen Ulnakopfgelenkfläche, grün: Distanz zwischen der türkisen und roten Parallelen ergibt die digital ausgemessene Ulnavarianz von $+2 \mathrm{~mm}$; radiologische Bestimmung der postoperativen Ulnavarianz jetzt mit marginaler Ulna-minus-Variante (Abb.1b) nach distaler Ulnaschaftverkürzungsosteotomie und dynamischer 6-Loch-Kompressionsplattenosteosynthese. Radiologische Bestimmung der DRUG-Gelenkflächenkonfiguration ( $b$ und $c$ in Abb. 1a) im Verhältnis zur Ulnaschaftlängsachse (a in Abb. 1a) nach Tolat [29] - hier Typ II mit schrägem Verlauf.

Tab. 2 Vergleich der Patienten mit Ulna-plus- bzw. Ulna-null- und Ulnaminus-Varianz: klinische Daten zu den Untersuchungszeitpunkten U0, U2 und U6.

\begin{tabular}{llccc} 
Merkmal & Zeitpunkt & „Ulna+“ & „Ulna-/0“ & p \\
Ruheschmerz & U0 & $5,20 \pm 2,94$ & $2,42 \pm 2,19$ & 0,019 \\
& U2 & $3,10 \pm 2,28$ & $1,33 \pm 2,19$ & 0,079 \\
& U6 & $1,00 \pm 2,31$ & $1,25 \pm 1,71$ & 0,774 \\
Belastungs- & U0 & $8,80 \pm 1,62$ & $7,75 \pm 1,86$ & 0,178 \\
schmerz & U2 & $7,60 \pm 2,27$ & $5,83 \pm 2,92$ & 0,135 \\
& U6 & $4,20 \pm 3,39$ & $3,33 \pm 3,50$ & 0,564 \\
Total_ROM_\% & U0 & $76,00 \pm 12,82$ & $74,25 \pm 11,10$ & 0,735 \\
& U2 & $77,40 \pm 13,76$ & $90,08 \pm 6,80$ & 0,011 \\
& U6 & $91,10 \pm 8,94$ & $93,42 \pm 6,99$ & 0,503 \\
Kraft\% & U0 & $52,60 \pm 18,66$ & $58,66 \pm 33,06$ & 0,595 \\
& U2 & $56,30 \pm 29,27$ & $85,75 \pm 36,24$ & 0,052 \\
& U6 & $77,00 \pm 21,05$ & $80,75 \pm 39,47$ & 0,780 \\
Mayo & U0 & $30,50 \pm 16,24$ & $40,00 \pm 26,11$ & 0,330 \\
& U2 & $38,00 \pm 24,06$ & $75,00 \pm 23,28$ & 0,002 \\
& U6 & $75,00 \pm 22,24$ & $78,75 \pm 27,23$ & 0,731 \\
Krimmer & U0 & $38,50 \pm 15,82$ & $46,25 \pm 21,96$ & 0,363 \\
& U2 & $45,00 \pm 21,86$ & $70,00 \pm 23,74$ & 0,019 \\
& U6 & $72,50 \pm 21,11$ & $74,17 \pm 29,68$ & 0,880 \\
\hline \multirow{2}{*}{ DASH } & U0 & $62,60 \pm 17,66$ & $46,25 \pm 21,44$ & 0,068 \\
& U2 & $57,90 \pm 15,08$ & $31,00 \pm 23,72$ & 0,006 \\
& U6 & $32,30 \pm 19,27$ & $28,50 \pm 24,50$ & 0,695
\end{tabular}

U0 = Ergebnis der Erstuntersuchung; U2 = Ergebnis der Untersuchung nach 2 Monaten; U6 = Ergebnis der Untersuchung nach 6 Monaten. Angegeben sind immer die Mittelwerte \pm Standardabweichung. Signifikanzniveau im Intersubjektvergleich: $\mathrm{p}=$ „Ulna + “vs. „Ulna- $/ 0$ “

stellt. Folgende Tests kamen zur Anwendung: Chi²-Test, gepaarter und unverbundener t-Test, Post-Hoc-Test. Des Weiteren erfolgten Prüfungen mit dem Levène-Test, dem Mauchly-Sphärizitätstest und eine Bonferroni-Korrektur. Bei kleinen Stichprobenumfängen wurde eine Greenhouse-Geisser-Korrektur vorgenommen. Ein p-Wert $<0,05$ wurde als statistisch signifikant gewertet. Zur Überprüfung der Effektivität der operativen Maßnahmen am TFCC in Abhängigkeit der präoperativen Ulnavarianz wurde das Gesamtkollektiv anschließend in 4 Gruppenver- gleichen auf statistisch signifikante Unterschiede zwischen den einzelnen Untergruppen (Intersubjektvergleich) und in den jeweiligen Untergruppen (Innersubjektvergleich) zu den jeweiligen Untersuchungszeitpunkten U0, U2 und U6 untersucht ( $\bullet$ Tab. 2-5). Im ersten Gruppenvergleich ( $\bullet$ Tab. 2) wurde das Gesamtkollektiv in Abhängigkeit der präoperativen Ulnavarianz in 2 Kohorten aufgeteilt: „Ulna + “ (Ulna-plus-Varianz, $\mathrm{n}=10$ ) vs. „Ulna-/0“ (Ulna-minus- bis Ulna-null-Varianz, $\mathrm{n}=12$ ). Im zweiten Gruppenvergleich ( $\diamond$ Tab. 3) wurde das Gesamtkollektiv in 3 Kohorten unterteilt und Patienten ohne UVO mit einer Palmer 2A- bzw. Palmer 2C-Läsion $(n=8)$ respektive einer Palmer 2D- bzw. Palmer 2E-Läsion $(n=5)$ mit Patienten mit einer Ulnaverkürzungsosteotomie ( $n=9)$ verglichen. Im dritten Gruppenvergleich ( $\bullet$ Tab. 4) wurden Patienten mit einer Ulna-plusVariante über 3 Millimetern $(n=4)$ mit Patienten mit einer Ulnaplus-Varianz von 3 Millimetern und darunter $(n=6)$ verglichen. Im vierten Gruppenvergleich ( $\bullet$ Tab. 5) wurde die Ulnavarianz nach UVO berücksichtigt. Patienten mit Ulna-minus-Variante (po_Ulna-; $n=4$ ) wurden mit Patienten mit Ulna-null-Variante (po_Ulna0; $n=2$ ) und Ulna-plus-Variante (po_Ulna+; $n=3$ ) verglichen. Präoperativ fand sich sowohl bezüglich der demografischen noch der klinischen Daten, abgesehen von den Schmerzen in Ruhe, kein statistisch relevanter Unterschied zwischen den Kohorten „Ulna + “ und „Ulna-/0“.

\section{Ergebnisse}

Zwischen den Kohorten „Ulna + “ und „Ulna-/0“ zeigte sich intraoperativ in Anlehnung an die Klassifikation der TFCC-Läsionen nach Palmer eine signifikant unterschiedliche Verteilung der degenerativen TFCC-Läsionen - siehe Patienten und Methoden $(\mathrm{p}<0,025)$.

In der Kohorte „Ulna + “ betrug die Ulnavarianz präoperativ im Durchschnitt $+3,1 \mathrm{~mm}$, in der Kohorte „Ulna-/0“ $-0,3 \mathrm{~mm}$ ( $p<0,001, \diamond$ Tab. 1). 9 der 10 Patienten mit einer Ulna-plus-Varianz bedurften aufgrund anhaltender Beschwerden bei der U2Untersuchung einer Ulnaverkürzung. Lediglich ein Patient mit 
Tab. 3 Vergleich der Patienten ohne UVO mit TFCC-Läsionen vom Typ $2 A+2 C(n=8)$ sowie Typ 2D+2E $(n=5)$ mit Patienten mit einer UVO ( $n=9)$.

\begin{tabular}{|c|c|c|c|c|c|c|c|}
\hline Merkmal & Zeitpunkt/ p (t) & „Palmer 2A+2C“ & $\mathbf{p}^{\prime}$ & „Palmer 2D+2E“ & $\mathbf{p}^{\prime}$ & „UVO“ & $\mathbf{p}^{*}$ \\
\hline \multirow[t]{6}{*}{ Ruheschmerz } & U0 & $2,50 \pm 2,39$ & 1 & $2,20 \pm 1,79$ & 0,08 & $5,56 \pm 2,88$ & 0,064 \\
\hline & (U0 vs. U2) & 1 & & 0,225 & & 0,031 & \\
\hline & U2 & $1,88 \pm 2,53$ & 0,52 & $0,20 \pm 0,45$ & 0,034 & $3,44 \pm 2,13$ & 0,409 \\
\hline & (U0 vs. U6) & 0,597 & & 1 & & 0,002 & \\
\hline & U6 & $1,25 \pm 1,91$ & 0,885 & $2,40 \pm 2,88$ & 0,188 & $0,33 \pm 1,00$ & 0,98 \\
\hline & (U2 vs. U6) & 1 & & 0,512 & & 0,02 & \\
\hline \multirow[t]{6}{*}{ Belastungsschmerz } & U0 & $7,75 \pm 1,58$ & 1 & $8,20 \pm 2,49$ & 1 & $8,67 \pm 1,66$ & 0,954 \\
\hline & (U0 vs. U2) & 0,589 & & 0,014 & & 0,186 & \\
\hline & U2 & $6,38 \pm 2,83$ & 1 & $5,40 \pm 3,13$ & 0,518 & $7,56 \pm 2,40$ & 1 \\
\hline & (U0 vs. U6) & 0,055 & & 0,233 & & 0,001 & \\
\hline & U6 & $3,13 \pm 3,31$ & 1 & $5,00 \pm 4,69$ & 1 & $3,56 \pm 2,88$ & 1 \\
\hline & (U2 vs. U6) & 0,194 & & 1 & & 0,015 & \\
\hline \multirow[t]{6}{*}{ Total_ROM_\% } & U0 & $76,63 \pm 11,44$ & 0,765 & $68,80 \pm 8,87$ & 0,654 & $77,11 \pm 13,08$ & 1 \\
\hline & (U0 vs. U2) & 0,046 & & 0,402 & & 1 & \\
\hline & U2 & $88,63 \pm 6,67$ & 1 & $85,20 \pm 18,46$ & 1 & $80,0 \pm 11,70$ & 0,474 \\
\hline & (U0 vs. U6) & 0,033 & & 0,062 & & 0,015 & \\
\hline & U6 & $92,88 \pm 8,53$ & 1 & $89,20 \pm 12,07$ & 0,989 & $93,67 \pm 3,99$ & 1 \\
\hline & (U2 vs. U6) & 0,364 & & 1 & & 0,053 & \\
\hline \multirow[t]{6}{*}{ Kraft \% } & U0 & $53,63 \pm 28,74$ & 1 & $63,80 \pm 39,12$ & 1 & $53,56 \pm 19,53$ & 1 \\
\hline & (U0 vs. U2) & 0,426 & & 0,566 & & 1 & \\
\hline & U2 & $79,88 \pm 36,57$ & 1 & $86,40 \pm 40,99$ & 0,489 & $57,89 \pm 30,58$ & 0,643 \\
\hline & (U0 vs. U6) & 0,245 & & 0,548 & & 0,047 & \\
\hline & U6 & $79,63 \pm 43,96$ & 1 & $75,40 \pm 34,40$ & 1 & $80,56 \pm 18,88$ & 1 \\
\hline & (U2 vs. U6) & 1 & & 1 & & 0,083 & \\
\hline \multirow[t]{6}{*}{ Mayo } & U0 & $43,75 \pm 28,00$ & 1 & $33,00 \pm 20,49$ & 1 & $30,00 \pm 17,14$ & 0,664 \\
\hline & (U0 vs. U2) & 0,113 & & 0,057 & & 0,62 & \\
\hline & U2 & $74,38 \pm 25,97$ & 1 & $68,00 \pm 25,40$ & 0,156 & $38,33 \pm 25,50$ & 0,028 \\
\hline & (U0 vs. U6) & 0,09 & & 0,081 & & 0,001 & \\
\hline & U6 & $79,38 \pm 29,45$ & 1 & $69,00 \pm 29,67$ & 1 & $79,44 \pm 18,28$ & 1 \\
\hline & (U2 vs. U6) & 1 & & 1 & & 0,002 & \\
\hline \multirow[t]{6}{*}{ Krimmer } & U0 & $45,63 \pm 19,90$ & 1 & $43,00 \pm 27,07$ & 1 & $40,00 \pm 16,01$ & 1 \\
\hline & (U0 vs. U2) & 0,145 & & 0,039 & & 1 & \\
\hline & U2 & $68,75 \pm 25,78$ & 1 & $65,00 \pm 25,74$ & 0,553 & $46,11 \pm 22,88$ & 0,22 \\
\hline & (U0 vs. U6) & 0,168 & & 0,171 & & 0,001 & \\
\hline & U6 & $73,13 \pm 32,29$ & 1 & $65,00 \pm 35,00$ & 1 & $78,33 \pm 10,90$ & 1 \\
\hline & (U2 vs. U6) & 1 & & 1 & & 0,008 & \\
\hline \multirow[t]{6}{*}{ DASH } & U0 & $48,00 \pm 21,81$ & 1 & $47,20 \pm 22,63$ & 0,62 & $62,33 \pm 18,70$ & 0,514 \\
\hline & (U0 vs. U2) & 0,428 & & 0,298 & & 1 & \\
\hline & U2 & $30,00 \pm 23,76$ & 1 & $38,80 \pm 26,86$ & 0,415 & $57,44 \pm 15,92$ & 0,051 \\
\hline & (U0 vs. U6) & 0,523 & & 0,578 & & 0,006 & \\
\hline & U6 & $29,75 \pm 25,40$ & 1 & $34,20 \pm 29,13$ & 1 & $28,44 \pm 15,83$ & 1 \\
\hline & (U2 vs. U6) & 1 & & 1 & & 0,004 & \\
\hline
\end{tabular}

U0= Ergebnis der Erstuntersuchung; U2 = Ergebnis der Untersuchung nach 2 Monaten; U6= Ergebnis der Untersuchung nach 6 Monaten. Angegeben sind immer die Mittel-

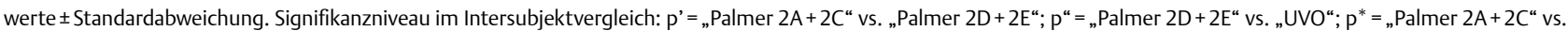
„UVO“. Signifikanzniveau im Innersubjektvergleich: $\mathrm{p}(\mathrm{t})$

einer Ulna-plus-Varianz von $1 \mathrm{~mm}$ fand sich durch das arthroskopische Débridement soweit gebessert, dass keine weitere Operation erforderlich war. Zum Zeitpunkt U6 waren 5 Ulnaverkürzungen knöchern konsolidiert (112,4 $\pm 37,1$ Tage nach UVO), 4 waren noch nicht knöchern durchbaut $(65,8 \pm 11,1$ Tage nach UVO). Das Ausmaß der Ulnaverkürzung betrug $3,6 \mathrm{~mm} \pm 2,0 \mathrm{~mm}$, postoperativ wurde eine Ulnavarianz von -0,2 (Min. -1, Max. 1) mm erreicht $(\mathrm{p}<0,001)$. Bei allen Patienten konnte die Ausgangssituation entweder durch ein alleiniges arthroskopisches Débridement des TFCC oder in Kombination mit der sekundären Ulnaverkürzung verbessert werden.

\section{Ruhe- und Belastungsschmerzen}

Die Schmerzen des Handgelenkes in Ruhe und bei Belastung verminderten sich postoperativ in der Gruppe „Ulna + " signifikant, wohingegen sich in der Gruppe „Ulna-/0“ lediglich der Belas- tungsschmerz zum Zeitpunkt U6 signifikant vermindert zeigte ( $\odot$ Tab. 2, $\odot$ Abb. 2, 3). Allerdings war der Ausgangswert der Ruheschmerzen bei Patienten mit einer Ulna-minus- bis Ulna-nullVarianz gering.

\section{Handgelenksbeweglichkeit}

Das Bewegungsausmaß des betroffenen Handgelenkes nahm in beiden Kohorten postoperativ signifikant zu ( $\bullet$ Tab. 2, $\odot$ Abb. 4).

\section{Kraft}

In Bezug auf die relative Kraft konnte weder in der Gruppe „Ulna+“ noch in der Gruppe „Ulna-/0“ ein signifikanter Unterschied zu den Zeitpunkten U2 und U6 festgestellt werden (॰ Tab.2, $\odot$ Abb. 5). 


\begin{tabular}{|c|c|c|c|c|}
\hline Merkmal & Zeitpunkt/p (t) & „Ulna > 3mm“ & „Ulna $\leq 3 \mathrm{~mm} “$ & $\mathbf{p}$ \\
\hline \multirow[t]{6}{*}{ Ruheschmerz } & U0 & $6,00 \pm 1,83$ & $4,67 \pm 3,56$ & 0,514 \\
\hline & (U0 vs. U2) & 0,239 & 0,174 & \\
\hline & U2 & $3,50 \pm 0,58$ & $2,83 \pm 2,99$ & 0,678 \\
\hline & (U0 vs. U6) & 0,021 & 0,645 & \\
\hline & U6 & $0 \pm 0$ & $1,67 \pm 2,86$ & 0,289 \\
\hline & (U2 vs. U6) & 0,004 & 1 & \\
\hline \multirow[t]{6}{*}{ Belastungsschmerz } & U0 & $9,25 \pm 0,58$ & $8,50 \pm 1,96$ & 0,506 \\
\hline & (U0 vs. U2) & 0,399 & 0,556 & \\
\hline & U2 & $7,50 \pm 1,73$ & $7,67 \pm 2,73$ & 0,917 \\
\hline & (U0 vs. U6) & 0,033 & 0,063 & \\
\hline & U6 & $5,25 \pm 0,50$ & $3,50 \pm 4,37$ & 0,457 \\
\hline & (U2 vs. U6) & 0,294 & 0,186 & \\
\hline \multirow[t]{6}{*}{ Total_ROM_\% } & U0 & $67,75 \pm 9,18$ & $81,50 \pm 12,43$ & 0,097 \\
\hline & (U0 vs. U2) & 0,078 & 1 & \\
\hline & U2 & $80,50 \pm 12,92$ & $77,33 \pm 15,10$ & 0,591 \\
\hline & (U0 vs. U6) & 0,06 & 0,348 & \\
\hline & U6 & $90,50 \pm 1,73$ & $91,50 \pm 11,90$ & 0,874 \\
\hline & (U2 vs. U6) & 0,751 & 0,082 & \\
\hline \multirow[t]{6}{*}{ Kraft\% } & UO & $42,25 \pm 9,47$ & $59,50 \pm 20,74$ & 0,163 \\
\hline & (U0 vs. U2) & 0,575 & 1 & \\
\hline & U2 & $54,50 \pm 6,35$ & $57,50 \pm 30,90$ & 0,885 \\
\hline & (U0 vs. U6) & 0,262 & 0,451 & \\
\hline & U6 & $77,00 \pm 19,75$ & $77,00 \pm 23,74$ & 1 \\
\hline & (U2 vs. U6) & 0,139 & 0,563 & \\
\hline \multirow[t]{6}{*}{ Mayo } & UO & $17,50 \pm 2,89$ & $39,17 \pm 15,63$ & 0,028 \\
\hline & (U0 vs. U2) & 0,403 & 1 & \\
\hline & U2 & $32,50 \pm 14,43$ & $41,67 \pm 29,61$ & 0,586 \\
\hline & (U0 vs. U6) & 0,026 & 0,047 & \\
\hline & U6 & $71,25 \pm 17,50$ & $77,50 \pm 26,22$ & 0,689 \\
\hline & (U2 vs. U6) & 0,159 & 0,085 & \\
\hline \multirow[t]{6}{*}{ Krimmer } & U0 & $33,75 \pm 15,82$ & $41,67 \pm 18,89$ & 0,471 \\
\hline & (U0 vs. U2) & 0,488 & 1 & \\
\hline & U2 & $47,50 \pm 11,90$ & $43,33 \pm 27,69$ & 0,787 \\
\hline & (U0 vs. U6) & 0,029 & 0,131 & \\
\hline & U6 & $72,50 \pm 6,46$ & $72,50 \pm 27,88$ & 1 \\
\hline & (U2 vs. U6) & 0,128 & 0,24 & \\
\hline \multirow[t]{6}{*}{ DASH } & UO & $72,50 \pm 7,3$ & $56,00 \pm 19,96$ & 0,158 \\
\hline & (U0 vs. U2) & 0,396 & 1 & \\
\hline & U2 & $58,50 \pm 10,47$ & $57,50 \pm 18,52$ & 0,925 \\
\hline & (U0 vs. U6) & 0,082 & 0,227 & \\
\hline & U6 & $33,00 \pm 16,27$ & $31,83 \pm 22,56$ & 0,932 \\
\hline & (U2 vs. U6) & 0,029 & 0,185 & \\
\hline
\end{tabular}

Tab. 4 Vergleich der Patienten mit einer Ulna-plus-Varianz präoperativ $>3 \mathrm{~mm}$ und $\leq 3 \mathrm{~mm}$.

\section{MMWS und Krimmer-Score}

Die objektive Einschätzung der Handgelenks- und Handfunktion mittels dem Modified Mayo Wrist- und dem Krimmer-Score ergab postoperativ einen signifikanten Anstieg der erzielten Punktwerte ( $\odot$ Tab. 2, $\odot$ Abb. 6, 7). Nach 6 Monaten konnten in der Kohorte „Ulna+“ 2 bzw. 3 der 10 Patienten ein gutes bzw. exzellentes Ergebnis im MMWS erzielen. In der Gruppe „Ulna10" waren es ein bzw. 6 der 12 Patienten mit einem gutem bzw. exzellentem Ergebnis (U6). 5 bzw. 3 Patienten zeigten in der Gruppe „Ulna+“ anhand des Krimmer-Scores eine gute bzw. sehr gute Handfunktion. In der Gruppe „Ulna-/0“ verfügten 7 Patienten über ein sehr gutes Ergebnis.

\section{DASH-Score}

Bei der subjektiven Einschätzung der Alltagseinschränkung erzielten Patienten mit einer Ulna-plus-Varianz zum Zeitpunkt U6 signifikant bessere Werte als präoperativ ( $\odot$ Tab. 2, $\odot$ Abb. 8). In der Gruppe „Ulna-/0“ konnte hingegen lediglich eine tendenzielle Verbesserung der Alltagseinschränkung festgestellt werden.

\section{Arbeitsfähigkeit}

Präoperativ waren 17 der 22 Patienten arbeitsunfähig, nach 6 Monaten waren es 4 Patienten ( $\bullet$ Tab. 1). Bei den 8 Patienten mit einer Ulna-plus-Varianz, die zum Zeitpunkt U0 arbeitsunfähig waren, bestand bei der Untersuchung nach 2 Monaten weiterhin Arbeitsunfähigkeit; bei der Abschlussuntersuchung nach 6 Monaten waren noch 2 Patienten weiterhin arbeitsunfähig. Von den 9 Patienten der Kohorte „Ulna-/0“, die zum Zeitpunkt U0 arbeitsunfähig waren, erlangten 7 bis zur Untersuchung nach 2 Monaten Arbeitsfähigkeit, die beiden anderen waren auch nach 6 Monaten nicht arbeitsfähig. 
Tab. 5 Vergleich der Patienten mit UVO in Abhängigkeit von der postoperativen Ulnavarianz.

\begin{tabular}{|c|c|c|c|c|c|c|c|}
\hline Merkmal & Zeitpunkt/p (t) & „po_Ulna+“ & $\mathbf{p}^{\prime}$ & „po_Ulna0“ & p“ & „po_Ulna-“ & $\mathbf{p}^{*}$ \\
\hline \multirow[t]{6}{*}{ Ruheschmerz } & UO & $3,00 \pm 2,65$ & 0,267 & $7,50 \pm 0,71$ & 1 & $6,50 \pm 2,65$ & 0,326 \\
\hline & (U0 vs. U2) & 0,551 & & 0,468 & & 0,351 & \\
\hline & U2 & $2,33 \pm 2,08$ & 1 & $5,50 \pm 0,71$ & 1 & $4,25 \pm 2,63$ & 0,912 \\
\hline & (U0 vs. U6) & 0,565 & & 0,127 & & 0,111 & \\
\hline & U6 & $0 \pm 0$ & 1 & $0 \pm 0$ & 1 & $0,75 \pm 1,50$ & 1 \\
\hline & (U2 vs. U6) & 0,575 & & 0,271 & & 0,464 & \\
\hline \multirow[t]{6}{*}{ Belastungsschmerz } & U0 & $8,00 \pm 2,00$ & 1 & $9,50 \pm 0,71$ & 1 & $8,75 \pm 1,89$ & 1 \\
\hline & (U0 vs. U2) & 0,809 & & 0,614 & & 1 & \\
\hline & U2 & $6,67 \pm 3,51$ & 1 & $6,50 \pm 0,71$ & 0,987 & $8,75 \pm 1,89$ & 0,923 \\
\hline & (U0 vs. U6) & 0,207 & & 0,211 & & 0,13 & \\
\hline & U6 & $3,67 \pm 3,22$ & 1 & $5,00 \pm 0$ & 1 & $2,75 \pm 3,59$ & 1 \\
\hline & (U2 vs. U6) & 0,365 & & 0,614 & & 0,13 & \\
\hline \multirow[t]{6}{*}{ Total_ROM_\% } & UO & $67,67 \pm 9,29$ & 1 & $71,00 \pm 9,90$ & 0,34 & $89,25 \pm 10,72$ & 0,134 \\
\hline & (U0 vs. U2) & 0,117 & & 0,509 & & 1 & \\
\hline & U2 & $76,33 \pm 6,66$ & 1 & $87,50 \pm 16,26$ & 1 & $79,00 \pm 13,98$ & 1 \\
\hline & (U0 vs. U6) & 0,079 & & 0,708 & & 0,909 & \\
\hline & U6 & $94,33 \pm 4,04$ & 0,398 & $89,00 \pm 0$ & 0,2 & $95,50 \pm 3,42$ & 1 \\
\hline & (U2 vs. U6) & 0,063 & & 1 & & 0,42 & \\
\hline \multirow[t]{6}{*}{ Kraft \% } & U0 & $65,67 \pm 30,07$ & 0,375 & $36,00 \pm 8,49$ & 0,95 & $53,25 \pm 6,19$ & 1 \\
\hline & (U0 vs. U2) & 1 & & 0,329 & & 1 & \\
\hline & U2 & $69,00 \pm 33,15$ & 1 & $59,00 \pm 2,83$ & 1 & $49,00 \pm 39,10$ & 1 \\
\hline & (U0 vs. U6) & 1 & & 0,1 & & 0,438 & \\
\hline & U6 & $73,33 \pm 22,81$ & 0,963 & $93,00 \pm 4,24$ & 1 & $79,75 \pm 21,00$ & 1 \\
\hline & (U2 vs. U6) & 1 & & 0,056 & & 0,483 & \\
\hline \multirow[t]{6}{*}{ Mayo } & U0 & $35,00 \pm 30,41$ & 0,981 & $17,50 \pm 3,54$ & 1 & $32,50 \pm 5,00$ & 1 \\
\hline & (U0 vs. U2) & 0,962 & & 0,377 & & 1 & \\
\hline & $\mathrm{U} 2$ & $45,00 \pm 39,69$ & 1 & $42,50 \pm 10,61$ & 1 & $31,25 \pm 22,87$ & 1 \\
\hline & (U0 vs. U6) & 0,139 & & 0,468 & & 0,054 & \\
\hline & U6 & $76,67 \pm 25,17$ & 1 & $77,50 \pm 17,68$ & 1 & $82,50 \pm 18,48$ & 1 \\
\hline & (U2 vs. U6) & 0,606 & & 0,991 & & 0,051 & \\
\hline \multirow[t]{6}{*}{ Krimmer } & U0 & $50,00 \pm 18,03$ & 0,485 & $27,50 \pm 10,61$ & 1 & $38,75 \pm 14,93$ & 1 \\
\hline & (U0 vs. U2) & 1 & & 0,377 & & 1 & \\
\hline & U2 & $53,33 \pm 22,55$ & 1 & $52,50 \pm 5,54$ & 1 & $37,50 \pm 29,58$ & 1 \\
\hline & (U0 vs. U6) & 0,172 & & 0,1 & & 0,14 & \\
\hline & U6 & $76,67 \pm 12,58$ & 1 & $75,00 \pm 7,07$ & 1 & $81,25 \pm 13,15$ & 1 \\
\hline & (U2 vs. U6) & 0,499 & & 0,211 & & 0,154 & \\
\hline \multirow[t]{6}{*}{ DASH } & UO & $51,33 \pm 27,21$ & 0,468 & $78,00 \pm 5,66$ & 1 & $62,75 \pm 12,01$ & 1 \\
\hline & (U0 vs. U2) & 1 & & 0,45 & & 1 & \\
\hline & U2 & $57,00 \pm 12,29$ & 1 & $53,00 \pm 14,14$ & 1 & $60,00 \pm 22,06$ & 1 \\
\hline & (U0 vs. U6) & 0,508 & & 0,37 & & 0,194 & \\
\hline & U6 & $33,00 \pm 16,09$ & 1 & $24,50 \pm 20,51$ & 1 & $27,00 \pm 17,93$ & 1 \\
\hline & (U2 vs. U6) & 0,128 & & 0,299 & & 0,288 & \\
\hline
\end{tabular}

U0 = Ergebnis der Erstuntersuchung; U2 = Ergebnis der Untersuchung nach 2 Monaten; U6 = Ergebnis der Untersuchung nach 6 Monaten. Angegeben sind immer die Mittelwerte \pm Standardabweichung. Signifikanzniveau im Intersubjektvergleich: p’= „po_Ulna + “vs. „po_Ulna0“; p“ = „po_Ulna0“ vs. „po_Ulna-“; p* = „po_Ulna + “vs. „po_Ulna-“. Signifikanzniveau im Innersubjektvergleich: $\mathrm{p}(\mathrm{t})$

Kohortenvergleich „Ulna+" vs. „Ulna-/0“ zum Zeitpunkt U2 und U6

2 Monate nach dem arthroskopischen Débridement bestanden signifikante Vorteile der Kohorte „Ulna-/0“ hinsichtlich der subjektiven Alltagseinschränkung („DASH“ $\mathrm{p}<0,01$ ), der Handgelenk- und Handfunktion (MMWS $\mathrm{p}<0,01$; Krimmer-Score $\mathrm{p}<0,025$ ) und des relativen Bewegungsausmaßes (Total_ROM_\% $\mathrm{p}<0,025)$. Bei der Betrachtung der relativen Kraft („Kraft\%“ $p>0,05)$, des Schmerzes in Ruhe (,Ruheschmerz“ $p>0,05)$ und des Schmerzes unter Belastung („Belastungsschmerz“ $p>0,05$ ) erreichte der Vorteil hingegen kein signifikantes Niveau (๑ Tab. 2). Zum Zeitpunkt U6 wiederum waren keine signifikanten Unterschiede von Schmerz, Kraft, Beweglichkeit, Alltagseinschränkung und Handfunktion vorhanden.
Behandlungsergebnisse abhängig vom Ausmaß der degenerativen Veränderungen

Das Gesamtkollektiv wurde zur Bewertung der arthroskopischen Behandlung der degenerativen TFCC-Läsionen in einem Gruppenvergleich in 3 Gruppen (,Palmer $2 \mathrm{~A}+2 \mathrm{C}$ “, $\mathrm{n}=8$; ,Palmer $2 \mathrm{D}+2 \mathrm{E}$ “ $\mathrm{n}=5$; „UVO“, n=9) unterteilt, die miteinander bezüglich der 7 Erhebungsmerkmale zu den 3 Zeitpunkten U0, U2 und U6 verglichen wurden ( $\bullet$ Tab. 3). Patienten mit einer höhergradigen, degenerativen Schädigung („Palmer $2 \mathrm{D}+2 \mathrm{E}^{\prime}$ ) wiesen nach 2 Monaten signifikant geringere Ruheschmerzen auf als die Gruppe „UVO“, die sich im Anschluss einer Ulnaverkürzung unterzog $(0,20 \pm 0,45$ bzw. 3,44 $\pm 2,13$ Punkte auf der visuellen Analogskala; $\mathrm{p}<0,05)$. Erwartungsgemäß wies die Gruppe mit geringgradiger, degenerativer Schädigung („Palmer $2 \mathrm{~A}+2 \mathrm{C}$ “) zum gleichen Zeitpunkt gegenüber der Gruppe „UVO“ eine signifikant bessere Handgelenkfunktion nach alleiniger arthroskopi- 


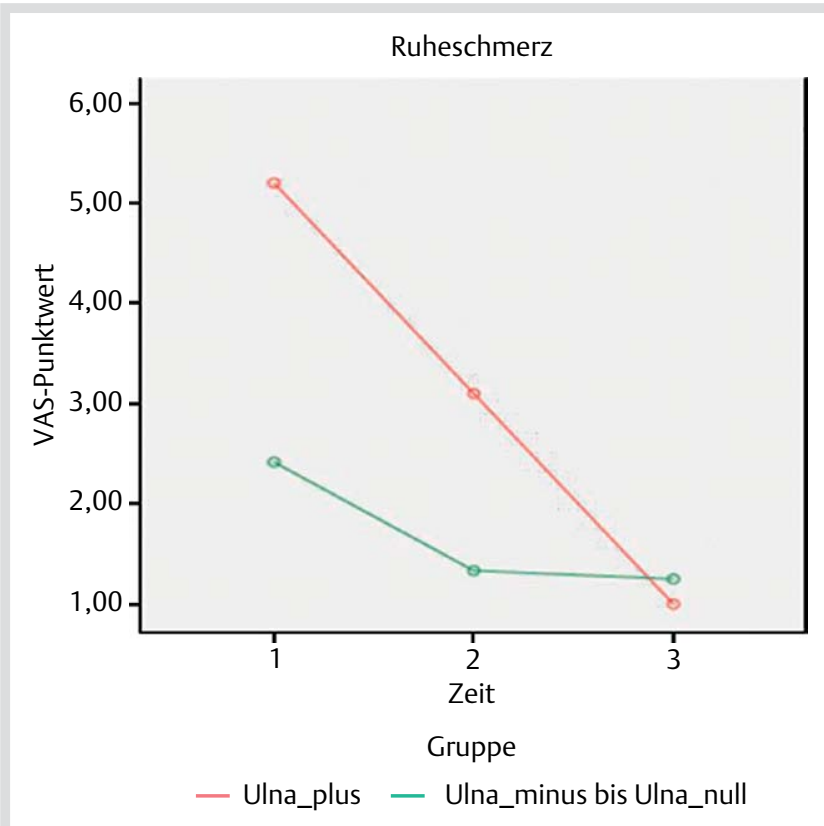

Abb. 2 Darstellung des subjektiven Schmerzempfindens des Handgelenkes in Ruhe (Ruheschmerz) anhand der Mittelwerte auf der visuellen Analogskala (VAS) von 0-10 präoperativ (1), 2 (2) und 6 (3) Monate postoperativ; grün: „Ulna-/0“, rot: „Ulna + “. Signifikanzniveau im Innersubjektvergleich: „Ulna + “ U0 vs. U2, $p=0,015$; U0 vs. U6, $p=0,038$; U2 vs. U6, $p=0,397$. „Ulna-/0“ U0 vs. U2, $p=0,469 ; \cup 0$ vs. U6, $p=0,235 ; U 2$ vs. $U 6, p=1,0$.

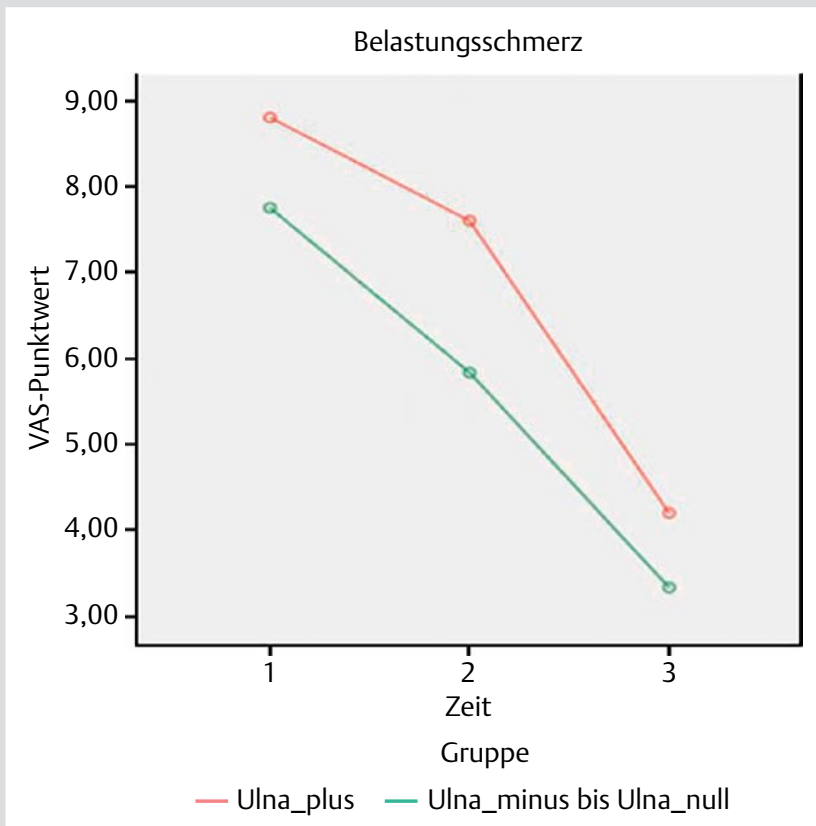

Abb. 3 Darstellung des subjektiven Schmerzempfindens bei Handgelenksbelastung (Belastungsschmerz) anhand der Mittelwerte auf der visuellen Analogskala (VAS) von 0-10 präoperativ (1), 2 (2) und 6 (3) Monate postoperativ; grün: „Ulna/0“, rot: „Ulna + “. Signifikanzniveau im Innersubjektvergleich: „Ulna + “ U0 vs. U2, $p=0,090$; U0 vs. U6, $p=0,002$; U2 vs. U6, $p=0,040$. „Ulna-/0“ U0 vs. U2, $p=0,054$; U0 vs. U6, $p=0,005$; U2 vs. U6, $p=0,155$.

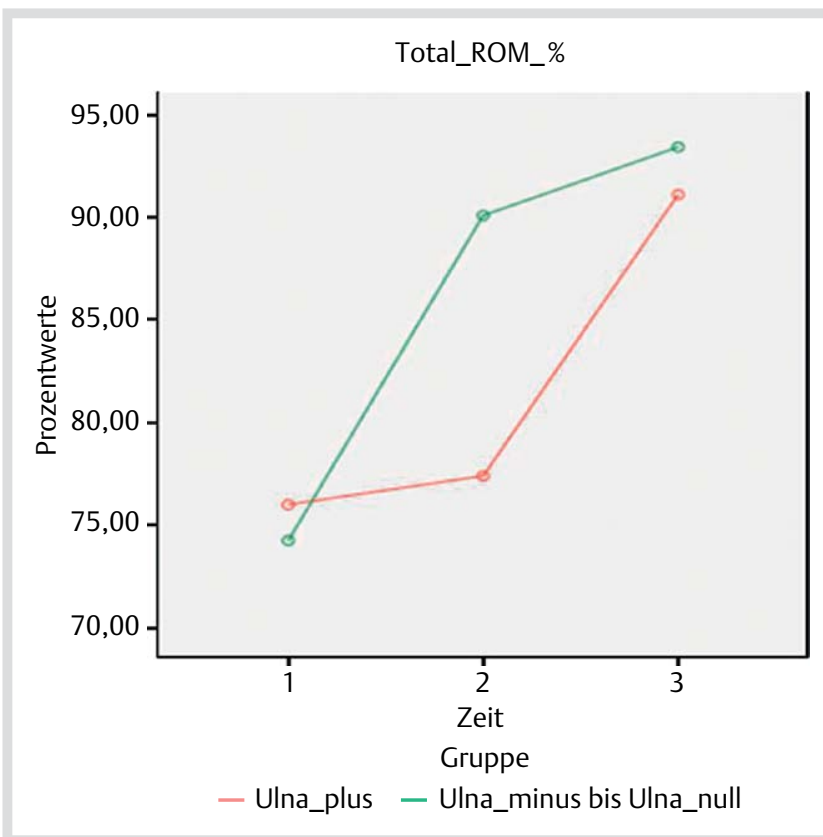

Abb. 4 Darstellung der relativen Bewegungsausmaß-Mittelwerte (Total_ROM_\%) zur Gegenseite in Prozent präoperativ (1), 2 (2) und 6 (3) Monate postoperativ; grün: „Ulna-/0“, rot: „Ulna+ “. Signifikanzniveau im Innersubjektvergleich: „Ulna + “ U0 vs. U2, $p=1,0 ; \cup 0$ vs. U6, $p=0,015$; U2 vs. U6, $p=0,026$. „Ulna-/0“ U0 vs. U2, $p=0,003$; U0 vs. U6, $p=0,001$; U2 vs. $U 6, p=0,347$.

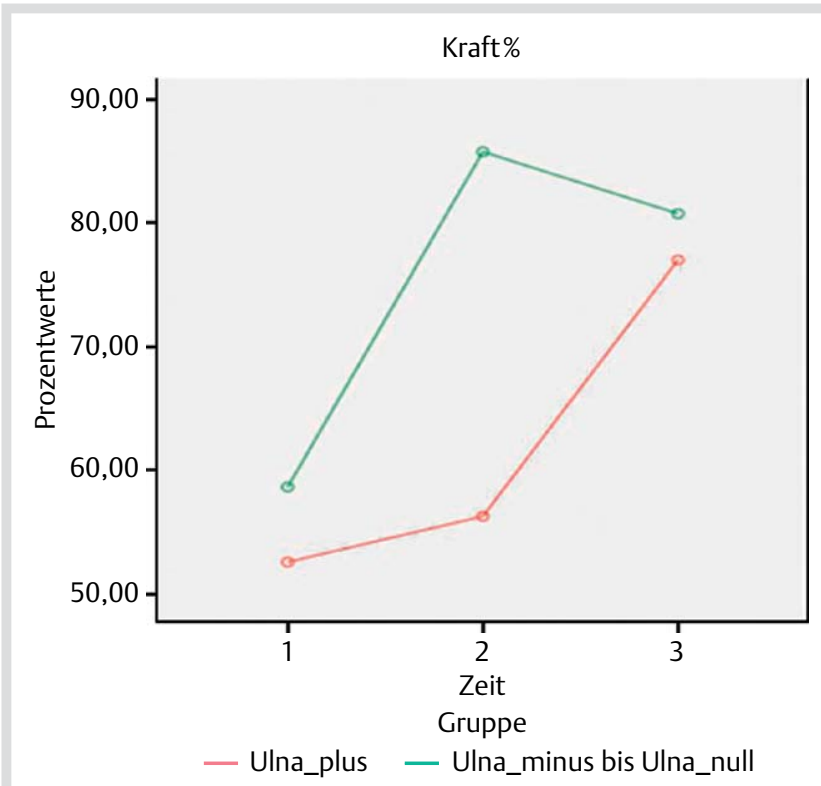

Abb. 5 Darstellung der relativen Kraft-Mittelwerte zur Gegenseite in Prozent (Kraft\%) präoperativ (1), 2 (2) und 6 (3) Monate postoperativ; grün: „Ulna-/0“, rot: „Ulna + “. Signifikanzniveau im Innersubjektvergleich: „Ulna + “ U0 vs. U2, $p=1,0 ; \cup 0$ vs. U6, $p=0,050$; U2 vs. U6, $p=0,079$. „Ulna-/0“ U0 vs. U2, $p=0,114 ; \cup 0$ vs. U6, $p=0,091 ; U 2$ vs. U6, $p=1,0$.

scher Behandlung der TFCC-Läsion auf (MMWS 74,38 $\pm 25,97$ Punkte bzw. 38,33 $\pm 25,50$ Punkte; $p<0,05$ ). Alle übrigen Parameter waren zwischen den 3 Gruppen nicht signifikant verschieden.

In der zeitlichen Entwicklung wiesen die Patienten der Gruppe „Palmer $2 \mathrm{~A}+2 \mathrm{C}$ “ eine signifikante Verbesserung in der Handgelenksbeweglichkeit auf. In der Gruppe „Palmer 2D+2E“ fand sich 


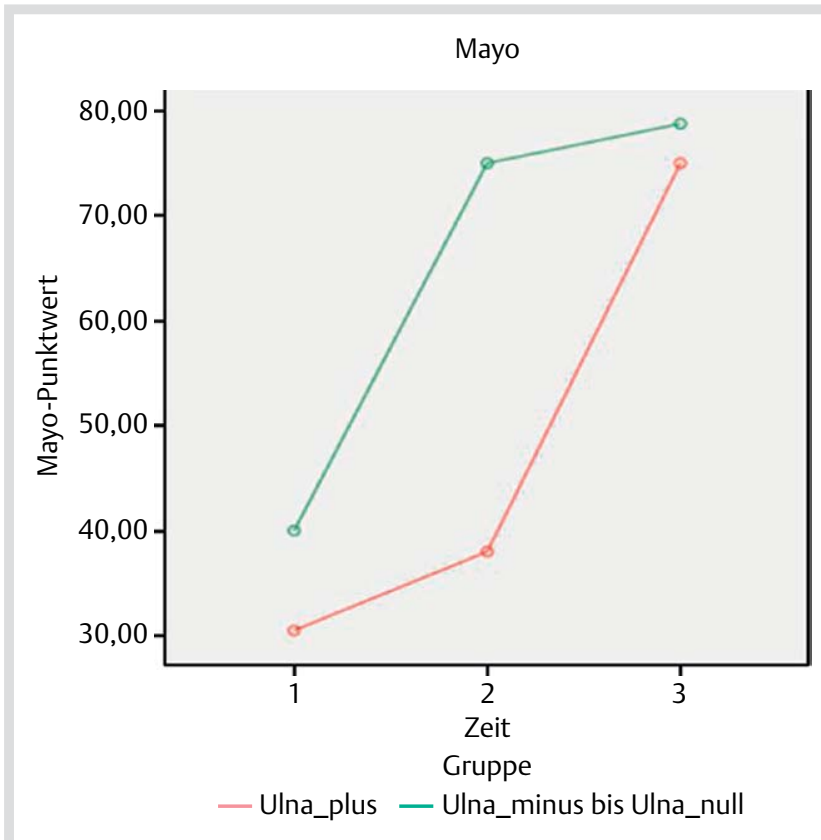

Abb. 6 Darstellung der Mayo-Score-Mittelwerte präoperativ (1), 2 (2) und 6 (3) Monate postoperativ; grün: „Ulna-/0“, rot: „Ulna + “. Signifikanzniveau im Innersubjektvergleich: „Ulna + “ U0 vs. U2, $p=0,615$; U0 vs. U6, $p=0,001 ;$ U2 vs. U6, $p=0,004$. „Ulna- $/ 0$ “ U0 vs. U2, $p=0,004$; U0 vs. U6, $p=0,003 ; U 2$ vs. U6, $p=1,0$.

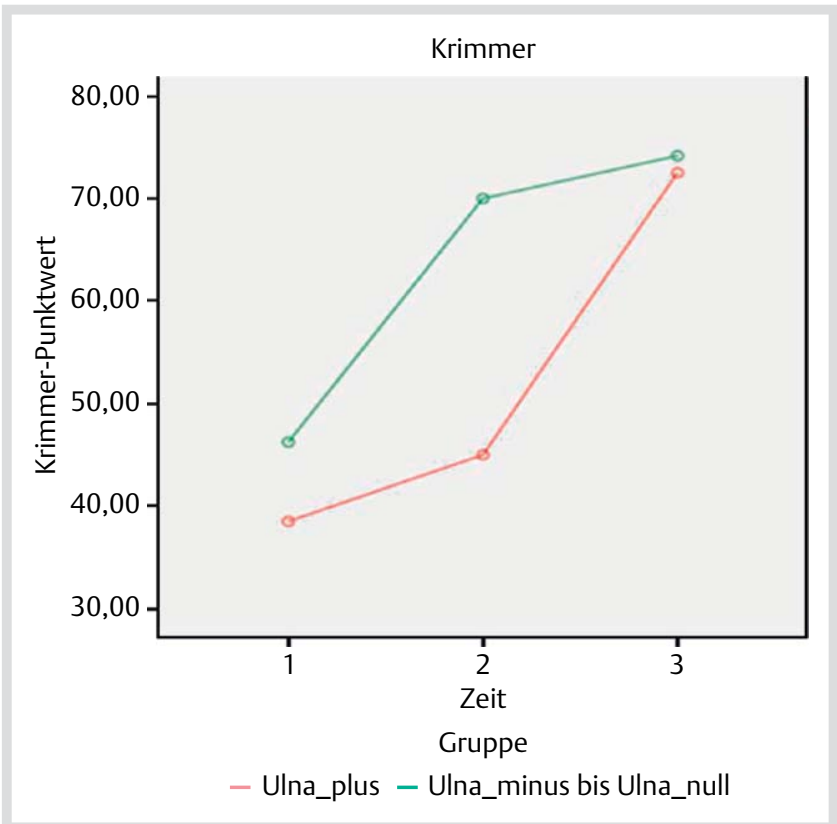

Abb. 7 Darstellung der Krimmer-Score-Mittelwerte präoperativ (1), 2 (2) und 6 (3) Monate postoperativ; grün: „Ulna-/0“, rot: „Ulna + “. Signifikanzniveau im Innersubjektvergleich: „Ulna + “ U0 vs. U2, $p=1,0 ; \cup 0$ vs. U6, $p=0,003$; U2 vs. U6, $p=0,025$. „Ulna- $/ 0$ “ U0 vs. U2, $p=0,012$; U0 vs. U6, $p=0,016 ;$ U2 vs. U6, $p=1,0$.

ein signifikanter Vorteil in Bezug auf den Belastungsschmerz und die Handgelenkfunktion. Bei den Patienten der Gruppe „UVO“ zeigten sich zahlreiche signifikante Unterschiede zwischen den Zeitpunkten U0 und U6, während solche zwischen UO und U2 nur einmalig vorlagen. Hier wurde das Signifikanzniveau erst zwischen den Zeitpunkten U2 und U6 erreicht: „Ruhe-

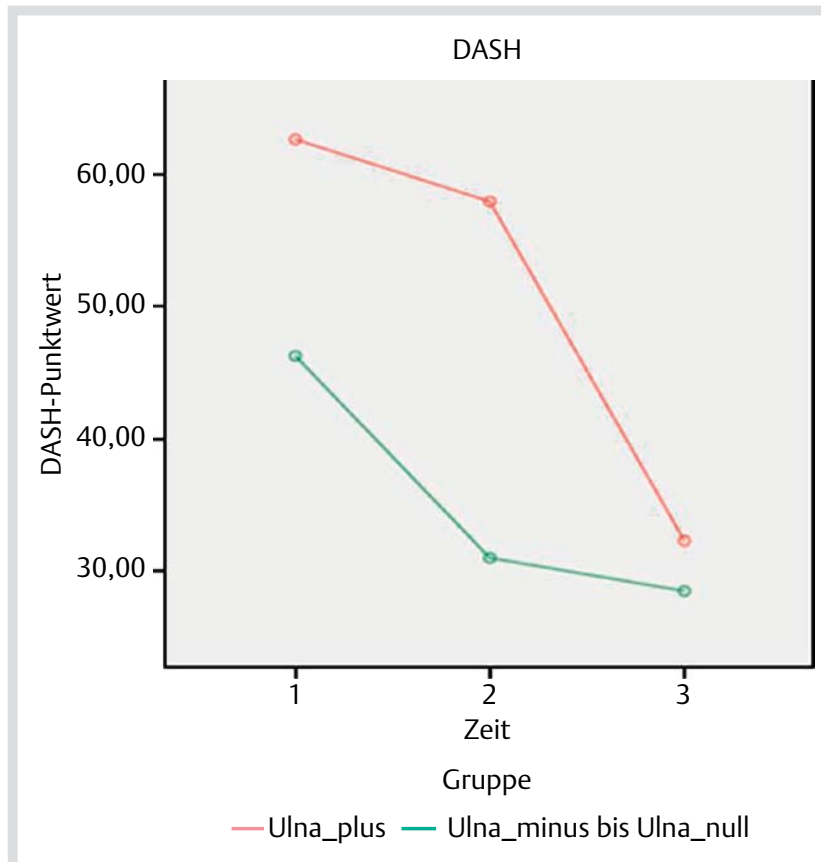

Abb. 8 Darstellung der DASH-Score-Mittelwerte präoperativ (1), 2 (2) und 6 (3) Monate postoperativ; grün: „Ulna-/0“, rot: „Ulna + “. Signifikanzniveau im Innersubjektvergleich: „Ulna + “ U0 vs. U2, $p=1,0$; $U 0$ vs. U6, $p=0,010 ; \cup 2$ vs. U6, $p=0,009$. „Ulna- $/ 0$ “ U0 vs. U2, $p=0,186 ; \cup 0$ vs. U6, $p=0,173 ;$ U2 vs. U6, $p=1,0$.

schmerz“, „Belastungsschmerz“, Kraft\%, „Total_ROM_\%“, „Mayo“, „Krimmer“ und „DASH“ ( $\bullet$ Tab. 3).

\section{Vergleich von Patienten mit einer präoperativen Ulna-} plus-Varianz $>3 \mathrm{~mm}$ und $\leq 3 \mathrm{~mm}$

Bei einer weiteren Analyse wurden 10 Patienten mit einer statischen Ulna-plus-Variante in 2 Gruppen „Ulna $>3 \mathrm{~mm}$ “ $(\mathrm{n}=4)$ bzw. „Ulna $\leq 3 \mathrm{~mm}$ “( $(n=6)$ unterteilt ( $\bullet$ Tab. 4). Zum Zeitpunkt U0 war in der Gruppe „Ulna $>3 \mathrm{~mm}$ “ die Handgelenksfunktion signifikant schlechter: „Mayo“-„Ulna > 3mm“ gegenüber „Mayo“„Ulna $\leq 3 \mathrm{~mm}$ “. Im Kollektiv „Ulna $\leq 3 \mathrm{~mm}$ “ bestand zwischen dem präoperativen Mayo-Punktwert (U0) und dem Mayo-Punktwert 6 Monate postoperativ (U6) ein signifikanter Vorteil, nachdem 5 der 6 Patienten einer Ulnaverkürzung unterzogen worden waren. Bei den Patienten des „Ulna > 3mm“-Kollektivs zeigten sich zahlreiche signifikante Unterschiede der Erhebungsmerkmale 6 Monate postoperativ (U6) und nach erfolgter Ulnaverkürzung gegenüber U0. Lediglich 2-mal bestand ein signifikanter Unterschied im Vergleich U2 und U6: „Ruheschmerz“ und „DASH“.

\section{Einfluss der Ulnavarianz nach Ulnaverkürzungsosteo-} tomie auf das klinische Ergebnis

Gleich, ob nach der UVO eine Ulna-plus-Varianz verblieb oder eine Ulna-null- bzw. Ulna-minus-Varianz erzielt wurde, fanden sich keine signifikanten Unterschiede bei den klinischen Ergebnissen ( $\bullet$ Tab. 5).

\section{Diskussion \\ $\nabla$}

Die vorliegende Studie wies Mängel auf. Die dynamische Ulnavarianz in der Belastungssituation wurde nicht berücksichtigt. Außerdem wurde das Mannerfelt-Ulrich intrinsic meter ${ }^{\circledR}$ zur 
Bestimmung der Handgelenksfunktion verwendet. Dieses Instrument bestimmt die Kraft des 3-Fingergriffs und nicht die des Grobgriffs. Darüber hinaus konnte im Rahmen der Studie in Ermangelung einer Kontrollgruppe nicht überprüft werden, ob die Patienten, die nach dem Untersuchungszeitraum U2 (2 Monate postoperativ) einer UVO zugeführt wurden, von einer längerfristigen Rekonvaleszenzphase nach Durchführung der Arthroskopie mit Fortsetzung der postoperativen Rehabilitation im Laufe der Zeit möglicherweise profitiert hätten, da die Patienten sehr frühzeitig auf die initial diskutierte UVO drängten. Ferner ist auf der einen Seite der kurze Nachuntersuchungszeitraum von 6 Monaten nach Durchführung der Handgelenksarthroskopie zu benennen, da infolge des kurzen Zeitraumes keine haltbaren Aussagen über das mögliche Auftreten von Spätkomplikationen nach UVO, wie eine Osteoarthritis im distalen Radioulnargelenk, die Notwendigkeit einer Materialentfernung oder eine Pseudarthrosenmanifestation, getroffen werden können. Eine Ergänzung der Studie ist geplant. Auf der anderen Seite erwies sich der kurze Nachuntersuchungszeitraum in Bezug auf die Patientenführung als vorteilhaft. Der gefürchtete Verlust von Patienten und deren Ergebnisse im postoperativen Verlauf mit Minderung der statistischen Power trat in keinem Fall auf. Darüber hinaus ist das prospektive Studiendesign mit klar definierten Ein- und Ausschlusskriterien als Stärke dieser Studie zu nennen.

Beeinflusst wird die ulnokarpale Krafteinleitung durch die Integrität des TFCC und die relative Ulnalänge im Verhältnis zur Radiuskonsole [4,21]. Bei neutraler Ulnavarianz (Ulna-null-Variante) und intaktem TFCC werden $18 \%$ der Kraft von der Hand über das ulnokarpale Gelenkkompartiment in den Unterarm eingeleitet [22]. Der TFCC übernimmt dabei einen relevanten Anteil der Kraftübertragung vom Karpus auf die Ulna [4]. Bei alleiniger Resektion des Discus triangularis wird die Kraftübertragung ulnokarpal um etwa 50\% $[8,13,16]$, bei kompletter Resektion des TFCC um 67\% des Ausgangswertes reduziert [22]. Die Stabilität des distalen Radioulnargelenkes mit dem Erhalt der radioulnaren und der ulnokarpalen Ligamente wird durch Belassen eines Resektionsrandes des TFCC gewährleistet $[11,13,16,17]$.

Die repetitive Kompression des TFCC zwischen den Gelenkpartnern führt zur allmählichen Degeneration des TFCC, von der die traumatische Rissbildung im jüngeren Lebensalter morphologisch abgegrenzt werden kann $[2,12,15,17]$. In der fünften und sechsten Lebensdekade findet sich der TFCC durchweg degenerativ verändert, in $40 \%$ ist er perforiert $[4,16,30,31]$. Läsionen des TFCC zählen zur häufigsten Indikation einer Handgelenksarthroskopie, wobei das arthroskopische Débridement in seiner Ausdehnung den individuellen Ansprüchen der Läsion gerecht werden muss [16,17]. Werden instabile Anteile des Discus ulnocarpalis bis zum Erreichen einer stabilen Resektionskante reseziert, ist eine ulnarseitige Beschwerdereduktion in bis $\mathrm{zu} 87 \%$ der Fälle beschrieben $[13,16]$. Andere Studien wiederum beschreiben unter dieser Therapie einen relevanten Anteil unzufriedener Patienten $[2,8,12,16,17]$. In der zusammenfassenden Betrachtung unserer beiden Kollektive des ersten Gruppenvergleichs spiegeln sich die kontroversen Literaturdaten wieder: Nach dem arthroskopischen Débridement der degenerativen TFCC-Läsion wiesen die 12 Patienten der Kohorte „Ulna-minus bis Ulna-null-Variante“ in allen gemessenen Parametern bis hin zur Lebensqualität eine Verbesserung auf. Dagegen blieben die 10 Patienten der Kohorte „Ulna-plus-Variante“ durch ein arthroskopisches TFCC-Débridement im Umfang ihrer Beschwerden annähernd unbeeinflusst. Dieser Umstand wird durch den zweiten Gruppenvergleich der vorliegenden Studie untermauert.
Bei allen Patienten konnte die Ausgangssituation entweder durch ein alleiniges arthroskopisches Débridement des TFCC oder in Kombination mit der sekundären UVO verbessert werden. Bei 21 von 22 Patienten erfolgte eine ulnokarpale Dekompression durch partielle Exzision des instabil flottierenden, zentralen Anteils des Discus ulnocarpalis, wobei sich die arthroskopische Dekompression bei 9 von 10 Patienten mit einer Ulnaplus-Variante in der Frühphase der Nachuntersuchung als insuffizient erwies. Nach der UVO kam es nahezu zur Angleichung aller Erhebungsmerkmale der Untergruppen „Ulna+“ und „Ulna-/0“. Tränkle et al. wiesen bei 29 Patienten nach UVO, von denen sich 18 Patienten im Vorfeld einem arthroskopischen Débridement des TFCC unterzogen hatten, eine Verbesserung der Handgelenksbeweglichkeit, der Kraft und der Schmerzen nach (12). Die Studien von Baek et al., Kim et al. und Fulton et al. untermauern mit ihren Ergebnissen die Feststellung einer Verbesserung der Beweglichkeit und der Kraft im Zuge der postoperativen Schmerzreduktion durch die ulnokarpal dekomprimierenden Maßnahmen $[2,5,24]$. Unsere Untersuchung unterstützt diese Feststellungen gleichermaßen, sodass eine konsekutive Verbesserung der Schmerzsymptomatik zu einer signifikanten Verbesserung der summierten Handgelenksbeweglichkeit und tendenziellen Kraftsteigerung der Hand geführt hat. Hierfür verantwortlich könnte die nachfolgende Freigabe von präoperativ gesperrten Bewegungsebenen des Handgelenkes durch die strukturellen, operativen Veränderungen im ulnokarpalen Kompartiment sein. Ferner könnte die Schmerzreduktion zu einer aktiv gesteigerten Bewegungsamplitude der betroffenen Handgelenke geführt haben. Die Bedeutung der postoperativen, physiotherapeutischen Behandlung ist ebenfalls zu diskutieren.

Eine etwaige Veränderung der nativ radiologisch erhobenen, relativen Ulnalänge in der Belastungssituation (posterioranteriorer Strahlengang des Handgelenkes und Grobgriff der Hand) zur Feststellung der dynamischen Ulnavarianz verblieb in der vorliegenden Studie unberücksichtigt. Gleichwohl stellt sich die Frage, ob die Patienten mit einem fortgeschrittenen Ulna-ImpaktionSyndrom, die keine UVO erhielten, von einer entsprechenden Versorgung profitiert hätten. Zur Klärung dieser Frage führten wir den zweiten Gruppenvergleich durch. Es bestanden tendenzielle Ergebnisunterschiede im Intersubjektvergleich der Gruppen, jedoch ohne Signifikanz. Aus dem alleinigen arthroskopischen Nachweis eines fortgeschrittenem Ulna-Impaktion-Syndroms ergibt sich nicht, dass Patienten in der statischen Ulnaminus- bis Ulna-null-Situation in dem Maße von einer UVO 2 Monate nach Durchführung eines arthroskopischen TFCC-Débridements profitieren können, wie die Patienten mit einer Ulnaplus-Variante.

Für Ulna-plus-Varianten von $1 \mathrm{~mm}$ bis $6 \mathrm{~mm}$ ist nach arthroskopischen Débridement einer TFCC-Läsion eine Schmerzlinderung bei einem exzellenten Mayo Modified Wrist Score berichtet [16]. Unsere Beobachtungen in der postoperativen Frühphase wiesen in der Ulna-plus-Situation gegensätzliche Ergebnisse mit erheblicher Patientenunzufriedenheit auf, die in der Konsequenz in einer frühzeitigen UVO mündeten. Dagegen sieht eine weitere Arbeit eine Abhängigkeit zwischen dem Erfolg des arthroskopischen Débridements stabiler TFCC-Läsionen und der Ulnavarianz: Bei Vorliegen einer Ulna-minus-Variante war kein zusätzlicher Eingriff notwendig, bei Ulna-null- und -plus-Varianten war in $22 \%$ bzw. $35 \%$ eine sekundäre Ulnaverkürzung erforderlich [32]. Schlechtere Resultate des Débridements in Begleitung einer Ulna-plus-Variante werden bestätigt und spezifiziert $[13,14]$. Der Vergleich des arthroskopischen Débridements des 
TFCC gegen eine isolierte Ulnaverkürzungsosteotomie und die Kombination beider Eingriffe zeigte signifikant bessere Ergebnisse sowohl für die Verkürzungsosteotomie als auch die Kombination der Eingriffe gegenüber dem isolierten Débridement in Bezug auf Handgelenksfunktion und -schmerzen [17]. Ein mehrzeitiges Vorgehen, ausgerichtet an der Ätiologie und den Begleitpathologien, wird als vorteilhaft diskutiert: So sollte bei einem Ulnavorschub und erhaltener Radiuskonsole ohne grobe Fehlstellung vor der Verkürzungsosteotomie ein arthroskopisches Débridement des TFCC ausgeführt werden und mögliche Begleitläsionen der Knorpelflächen bzw. der karpalen Bänder nachgewiesen sowie therapiert werden [12,32]. Nach Auffassung vieler Autoren bietet die UVO gegenüber anderen ulnokarpal druckentlastenden Verfahren wie der distalen Ulnaresektion (Wafer) und der Dekompressionsosteotomie des Ulnakopfes (Pechlaner) als extraartikuläres Verfahren Vorteile, obgleich die postoperativen Risiken einer Ulnaschaftpseudarthrose, einer sekundären Osteoarthritis des DRUG oder eines empfindlichen Plattenlagers mit Notwendigkeit einer operativen Materialentfernung bekannt sind $[4,5,11,12,24,32]$. So bleibt die Integrität des TFCC, des ulnokarpalen Gelenkkompartimentes sowie des DRUG unberührt, neben der ulnokarpalen Druckerhöhung wird ein mögliches stylokarpales Impaktion adressiert, milde Instabilitäten der ulnokarpalen Säule und des DRUG werden durch Spannungszunahme des TFCC stabilisiert und Resektionsausmaße über $4 \mathrm{~mm}$ sind im Vergleich zur Wafer-Prozedur problemlos möglich $[4,5,11,12,24,32]$. In unserer Studie wiesen nach UVO und angestrebter postoperativer Ulna-null- bzw. Ulna-minus-Variante bis zu einem Millimeter 3 Patienten weiterhin eine Ulna-plus-Variante von einem Millimeter auf, jedoch konnte dieser Umstand im vierten Gruppenvergleich keinen statistisch signifikanten Einfluss auf die subjektiv empfundenen Beschwerden und Alltageinschränkungen sowie auf die objektiv erhobenen Funktionsparameter aufzeigen. Einschränkend anzumerken ist hierbei die geringe Gruppengröße und die daraus resultierende, geringe Aussagekraft (Power) des Gruppenvergleichs.

Darüber hinaus zeigte sich in unserem Kollektiv, dass die Patienten, die sich in der Ulna-plus-Situation einer sekundären UVO unterzogen und eine präoperative Ulna-plus-Variante von über 3 Millimetern aufwiesen, klinisch von der kombinierten ulnokarpalen Dekompression profitierten und sich zur Ausgangssituation signifikant verbesserten. Die Patienten mit einer Ulnaplus-Variante von 3 Millimetern und darunter wiesen ebenfalls klinische Verbesserungen auf, wobei bis auf das Merkmal „Mayo“ alle weiteren Unterschiede tendenzieller Natur waren. Insgesamt unterschieden sich die postoperativen Ergebnisse beider Gruppen in der Gegenüberstellung unwesentlich und erreichten daher kein Signifikanzniveau, sodass geschlossen werden kann, dass Patienten mit einer präoperativen Ulna-plus-Variante von über 3 Millimetern und gescheitertem arthroskopischen Débridement genauso von einer UVO profitieren wie die Patienten mit einer präoperativen Ulna-plus-Variante von 3 Millimetern und weniger.

Vergleichbare Verlaufsergebnisse liegen in prospektiver Erhebung nicht vor, insbesondere subjektive Ergebnisse werden in älteren Arbeiten unzureichend berücksichtigt, sodass die Diskussion national und international nicht abgeschlossen, eine Evidenz gestützte Therapieempfehlung nicht verabschiedet ist $[6,14]$. Auch in unserer Studie verbleibt die offensichtliche Notwendigkeit den unter der Ulna-plus-Situation kompromittierten TFCC nach arthroskopischem Débridement zu entlasten. Der an dieser Stelle wiederholt in die Diskussion eingebrachte Faktor Alter spielte wenigstens in unserem Kollektiv statistisch keine Rolle [16,30-32].

\section{Schlussfolgerung}

\section{$\nabla$}

Degenerative Läsionen des TFCC können im Falle einer Ulnaminus- und Ulna-null-Variante durch ein arthroskopisches Débridement erfolgreich behandelt werden. Ulna-plus-Varianten von 2 Millimeter und mehr können bei Beschwerdepersistenz nach einem gescheiterten Débridement des TFCC sekundär erfolgreich mit einer Ulnaverkürzung 2 Monate postoperativ kombiniert werden.

\section{Danksagung \\ $\nabla$}

Ich möchte mich beim gesamten Team der PHW-Abteilung im Klinikum Nordstadt und allen anderen Wegbegleitern bedanken, die mich bei der Projektarbeit unterstützt haben. Ein besonderer Dank gilt Frau Bettina Gleissner, die als Abteilungssekretärin durch ihren unermüdlich koordinierenden und vorbereitenden Einsatz die Untersuchungen der Patienten reibungslos ermöglicht hat.

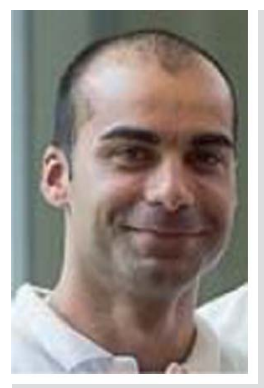

Giuseppe Broccoli

Geb. am 06.10.1977 in Neustadt a. Rbge.

1998-2005 Medizinstudium an der

Medizinischen Hochschule Hannover.

2005 Approbation in Deutschland,

Schweden und Norwegen. 2005-2007

Assistenzarzt der Chirurgischen Klinik,

Klinikum Großburgwedel (Chefärzte Dr.

med. H.-E. van Alste/Dr. med. A. Elsen/PD

Dr. med. J. Mall). 2008-2010 Assistenz-

arzt der Klinik für Unfallchirurgie und Orthopädie, Klinikum Nordstadt Hannover (Dr. med. K. Westermann/PD Dr. med.

J. Isenberg). 2010 Assistenzarzt der Abteilung für Plastische, Ästhetische und Handchirurgie, Klinikum Nordstadt Hannover (Chefarzt Dr. med. L. Müller). 2011 Assistenzarzt der Klinik für Orthopädie, Unfallchirurgie und Sportmedizin, Klinikum Agnes-Karll Laatzen, (Prof. Dr. med. Rühmann/ Dr. Berndt). 2012 Facharztanerkennung im Gebiet Orthopädie und Unfallchirurgie. Seit 2012 Facharzt in der Abteilung für Plastische, Ästhetische und Handchirurgie, Klinikum Nordstadt Hannover (Dr. med. L. Müller). Kumulative Promotionsarbeit bei PD Dr. med. J. Isenberg, Klinik für Unfallchirurgie und Orthopädie, Klinikum Nordstadt Hannover.

\section{Interessenkonflikt: Nein}

\section{Literatur}

1 Hempfling $H$. Die Läsionen des Handgelenkdiskus. Pathologie, Diagnostik und Therapie. Orthopäde 2004; 33: 657-675

$2 \mathrm{Kim}$ BS, Song HS. A comparison of ulnar shortening osteotomy alone versus combined arthroscopic triangular fibrocartilage complex debridement and ulnar shortening osteotomy for ulnar impaction syndrome. Clin Orthop Surg 2011; 3: 184-190

3 Friedman SL, Palmer AK. The ulnar impaction syndrome. Hand Clin 1991; 7: 295-310

4 Sammer DM, Rizzo M. Ulnar impaction. Hand Clin 2010; 26: 549-557 
5 Fulton C, Grewal R, Faber KJ et al. Outcome analysis of ulnar shortening osteotomy for ulnar impaction syndrome. Can J Plast Surg 2012; 20: e1-e5

6 Schädel-Höpfner M, Müller K, Gehrmann S et al. Therapie von Läsionen des „triangular fibrocartilage complex“. Unfallchirurg 2012; 115 : 582-588

7 Cha SM, Shin HD, Kim KC. Positive or negative ulnar variance after ulnar shortening for ulnar impaction syndrome: a retrospective study. Clin Orthop Surg 2012; 4: 216-220

8 Palmer AK, Werner FW, Glisson RR et al. Partial excision of the triangular fibrocartilage complex. J Hand Surg Am 1988; 13: 391-394

9 Palmer AK. Triangular fibrocartilage complex lesions: a classification. J Hand Surg Am 1989; 14: 594-606

10 Ewert A, Mittlmeier T. Indikation und Technik der Handgelenkarthroskopie. Orthopäde 2007; 36: 1157-1168

11 Beyermann K, Krimmer H, Lanz U. TFCC-Läsionen. Diagnostik und Therapie. Orthopäde 1999; 28: 891-898

12 Tränkle $M$, van Schoonhoven J, Krimmer $H$ et al. Indikation und Ergebnisse der Ulnaverkürzungsosteotomie bei ulnokarpalem Handgelenkschmerz. Unfallchirurg 2000; 103: 197-202

13 Minami A, Ishikawa J, Suenaga $N$ et al. Clinical results of treatment of triangular fibrocartilage complex tears by arthroscopic debridement. J Hand Surg Am 1996; 21: 406-411

14 Minami A, Kato $\mathrm{H}$. Ulnar shortening for triangular fibrocartilage complex tears associated with ulnar positive variance. J Hand Surg Am 1998; 23: 904-908

15 Sachar K. Ulnar-sided wrist pain: evaluation and treatment of triangular fibrocartilage complex tears, ulnocarpal impaction syndrome, and lunotriquetral ligament tears. J Hand Surg Am 2008; 33: 1669-1679

16 Husby T, Haugstvedt JR. Long-term results after arthroscopic resection of lesions of the triangular fibrocartilage complex. Scand J Plast Reconstr Surg Hand Surg 2001; 35: 79-83

17 Tan SW, Ng SW, Tan SH et al. Arthroscopic debridement of intercarpal ligament and triangular fibrocartilage complex tears. Singapore Med J 2012; 53: 188-191
18 Saffar $P$. The pathology of the long ulna: anatomy and treatment. J Hand Surg Eur Vol 2007; 32: 608-619

19 Tatebe M, Horii E, Nakao E et al. Repair of the triangular fibrocartilage complex after ulnar-shortening osteotomy: second-look arthroscopy. J Hand Surg Am 2007; 32: 445-449

20 Watanabe A, Souza F, Vezeridis PS et al. Ulnar-sided wrist pain. II. Clinical imaging and treatment. Skeletal Radiol 2010; 39: 837-857

21 Palmer AK, Glisson RR, Werner FW. Relationship between ulnar variance and triangular fibrocartilage complex thickness. J Hand Surg Am 1984; 9: 681-682

22 Palmer AK, Werner FW. Biomechanics of the distal radioulnar joint. Clin Orthop Relat Res 1984; 187: 26-35

23 Gelberman RH, Salamon PB, Jurist JM et al. Ulnar variance in Kienböck's disease. J Bone Joint Surg Am 1975; 57: 674-676

24 Baek GH, Lee HJ, Gong HS et al. Long-term outcomes of ulnar shortening osteotomy for idiopathic ulnar impaction syndrome: at least 5-years follow-up. Clin Orthop Surg 2011; 3: 295-301

25 Whipple TL, Marotta JJ, Powell JH $3^{\text {rd }}$. Techniques of wrist arthroscopy. Arthroscopy 1986; 2: 244-252

26 Atzei A, Luchetti R. Foveal TFCC tear classification and treatment. Hand Clin 2011; 27: 263-272

27 Outerbridge RE. The etiology of chondromalacia patellae. J Bone Joint Surg Br 1961; 43-B: 752-757

28 Geissler WB, Freeland AE, Savoie FH et al. Intracarpal soft-tissue lesions associated with an intra-articular fracture of the distal end of the radius. J Bone Joint Surg Am 1996; 78: 357-365

29 Tolat AR, Sanderson PL, De Smet L et al. The gymnast's wrist: acquired positive ulnar variance following chronic epiphyseal injury. J Hand Surg Br 1992; 17: 678-681

30 Mikić ZD. Age changes in the triangular fibrocartilage of the wrist joint. J Anat 1978; 126: 367-384

31 Viegas SF, Patterson RM, Hokanson JA et al. Wrist anatomy: incidence, distribution, and correlation of anatomic variations, tears, and arthrosis. J Hand Surg Am 1993; 18: 463-475

32 Beyermann K, Busse F, Krimmer H et al. Arthroskopisches Debridement bei Läsionen des TFCC. Arthroskopie 2002; 15: 21-25 